\title{
Experiential Metaphors in Abstract Games Jason Begy
}

Concordia University, Department of Communication Studies 7141 Sherbrooke Street West, CJ 3.230, 3rd Floor Montreal, QC H4B 2Y1, Canada jsbegy@gmail.com

\section{INTRODUCTION}

As artifacts, abstract games offer uncommon barriers to criticism.

These games often appear to be little more than sets of seemingly arbitrary symbols or shapes that are manipulated or transformed according to equally arbitrary rules, and it can be difficult to see these games as anything but interesting little challenges. Part of this difficulty stems from the fact that these games are not obviously about someone or something in the way other media forms - including other gamesare. For Ian Bogost (2009), this lack of "aboutness" - meaning whether the game is clearly about an idea, concept, or theme-is a major barrier to interpretation:

Can we talk about such games the way we talk about, say, BioShock or Pac-Man or SimCity? All of those games offer aboutness of some kind, whether through narrative, characterization, or simulation. In each, there are concrete topics that find representation in the rules and environments. Indeed, it's hard to talk about abstract games precisely because they are not concrete. Those with more identifiably tangible themes offer some entry point for thematic interpretation.

In this paper, I argue that one entry point for interpretation of an abstract game is the experience of playing it, via experiential metaphor (Rusch 2009). After defining “abstract game," I discuss the theory behind experiential metaphors and offer two examples of how they can 
be used in criticism of abstract games. The goal of this critical method is to provide a new means for game players to construct their own interpretations of a game; the emphasis throughout is on what the player experiences and how it relates to his or her own life.

Here I am using "metaphor" not in the sense of a rhetorical or linguistic flourish, but rather in the cognitive sense as employed by Lakoff and Johnson (1980) and Johnson (1987). Metaphorical projection is the act of applying knowledge or experience from one area of experience to another. Following Lakoff and Johnson (1980), I will refer to the domain that knowledge is taken from as the "source domain" and the domain to which it is applied as the "target domain."

\section{ABSTRACT GAMES}

For purposes of this paper, I define abstract game as follows: Abstract games are those in which the game objects are not signs in the game's fiction, or, if they are, they operate primarily in the symbolic mode. This definition contains three key elements: game objects, rules and fiction, and Peircean sign modalities. The remainder of this section defines these concepts.

\section{Definition}

By game object I mean a significant, isolatable entity that influences or modifies other entities within the game. For example, Mario of Super Mario Bros. (Nintendo 1985) fame is typically a game object in that he can influence other objects, such as "goombas" (by stomping on them) or coins (by collecting them). Mario's mustache is typically not a game object, nor is the person playing the game; in board games the term refers to the actual pieces being manipulated by the player. Game objects generally fall under Järvinen's (2008) category of "components," though here I intentionally define the term broadly to allow for general discussion of the elements that comprise a game. The second component of the definition is Juul's theory of game rules 
and fiction (2005). Juul defines rules as follows:

Rules specify limitations and affordances. They prohibit players from performing actions and this affords players meaningful actions that were not otherwise available; rules give games structure. The board game needs rules that let the players move their pieces as well as preventing them from making illegal moves; the video game needs rules that let the characters move as well as rules that prevent the character from reaching the goal immediately.

Fiction refers to the world in which a game takes place:

$[\mathrm{M}]$ ost video games also project a fictional world: The player controls a character; the game takes place in a city, in a jungle, or anywhere else. Such fictional game worlds, obviously, do not actually exist; they are worlds that the game presents and the player imagines (2005).

This distinction is significant, as I will be discussing the roles game objects play within a game's rules and its fiction.

The third component of the definition is Peircean sign modalities. For Peirce, signs are comprised of three elements: the representamen (the form the sign takes), the interpretant (how the sign is interpreted), and the object (that which the sign refers to). Signs operate in three different modes: symbolic, iconic, and indexical. These modes are not mutually exclusive, and any given sign can operate in any combination or number of modes. In the symbolic mode the representamen (or signifier) does not resemble the object; rather, their relationship is "arbitrary or conventional" (Chandler 1997). Symbols "have become associated with their meanings by usage" (Peirce 1998). As examples, Peirce offers "most words, and phrases, and speeches, and books, and libraries" (1998). In the iconic mode the representamen "is perceived 
as resembling or imitating the [object]" (Chandler 1997). Iconic signs "serve to convey ideas of the things they represent simply by imitating them" (Peirce 1998). Portraits, cartoons, onomatopoeia, and imitative gestures are examples of icons (Chandler 1997). In the third mode, indexical, the representamen is "not arbitrary, but is directly connected in some way (physically or causally) to the [object]." Indexical signs "show something about things, on account of their being physically connected with them" (Peirce 1998). Examples include "natural signs" such as smoke, thunder, and footprints, as well as measuring devices such as thermometers and clocks" (Chandler 1997).

All signs function within codes, which are "a framework within which signs make sense" (Chandler 1997). For example, we understand the meaning of a written word only if we have access to the relevant code, that is, the language in which the word is written. When game objects are treated as signs, the rules of the game act as one code in which the sign is situated. The following examples assume that the observer understands the relevant codes, which include the rules of the game as well as cultural codes.

\section{Application}

Game objects can operate as signs that signify through both the game fiction and the rules. I will use the term fiction-sign when considering how the game object operates as a sign in the game's fiction, and rules-sign when considering how it operates as a sign in the rules. To demonstrate how this functions, consider a rook taken from a chess set, such as the one in Figure 1.

Figure 1: A rook is a sign in terms of game fiction and game rules.

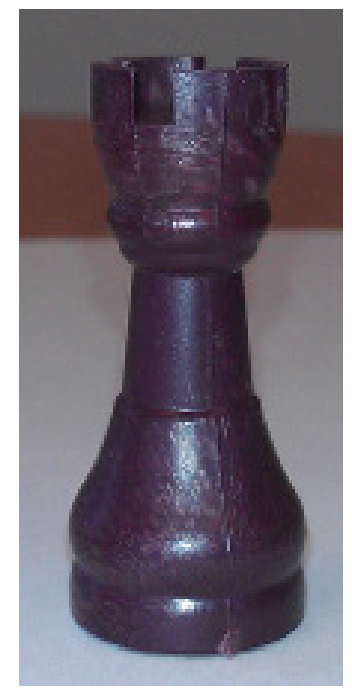


If the rook is considered as a fiction-sign, the representamen is the rook itself, and the object is a castle, or a tower. This particular sign operates primarily as an icon, as it resembles an actual castle. However, as a rules-sign the rook's primary modality changes. The representamen is still the rook itself, but the object is the set of rules governing the rook's in-game behavior. Because there is no connection between the form of the rook and how it behaves - castles do not typically move- the rules-sign is symbolic. Thus, objects function as signs on both levels. My definition of abstract game relies on the modality of objects as fiction-signs. Most other chess pieces are iconic fiction-signs. The knight typically takes the form of a horse, while the bishop features a clerical hat. The queen and king are both depicted wearing crowns, indicating their royal status. The pawn is traditionally the least iconic: taken by itself, it does not seem to represent anything. It is, however, appropriately diminutive compared with the other pieces, and the traditional sphere at the top of the piece can be said to resemble a head. In this instance, knowing the code causes the sign to operate more in the iconic mode. Because the objects are predominantly iconic fiction-signs, chess is not an abstract game.

In contrast, consider the go stones in Figure 2.

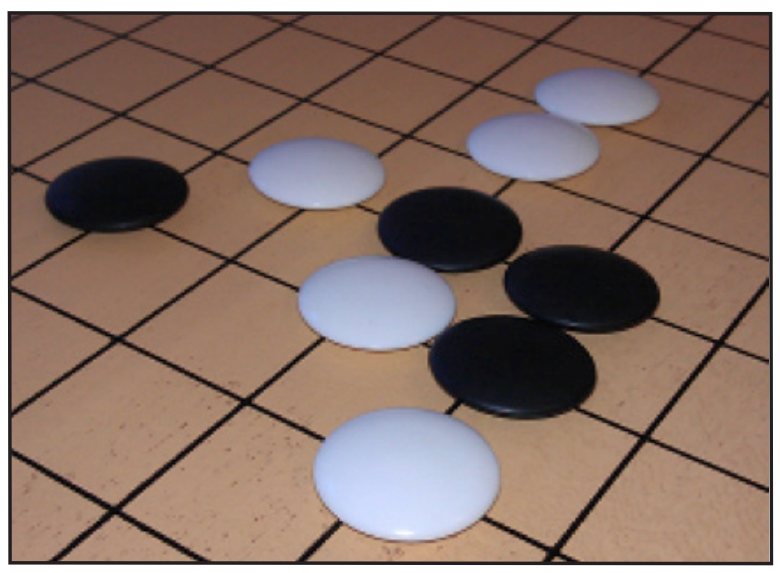

Figure 2: Go stones during a game. 
As with the rook, these stones act as rules-signs. The representamen is the stone itself, and the object is the set of rules governing its behavior. Because there is no connection between the form of the sign and the rules, the rules-sign is symbolic. But if the stones are taken as fiction-signs, it becomes apparent that they are not signs at all: they are simply stones that do not represent anything. Thus, go is an abstract game because its objects do not function as fiction-signs.

A third category can be found in Rod Humble's The Marriage (2007), shown in Figure 3.

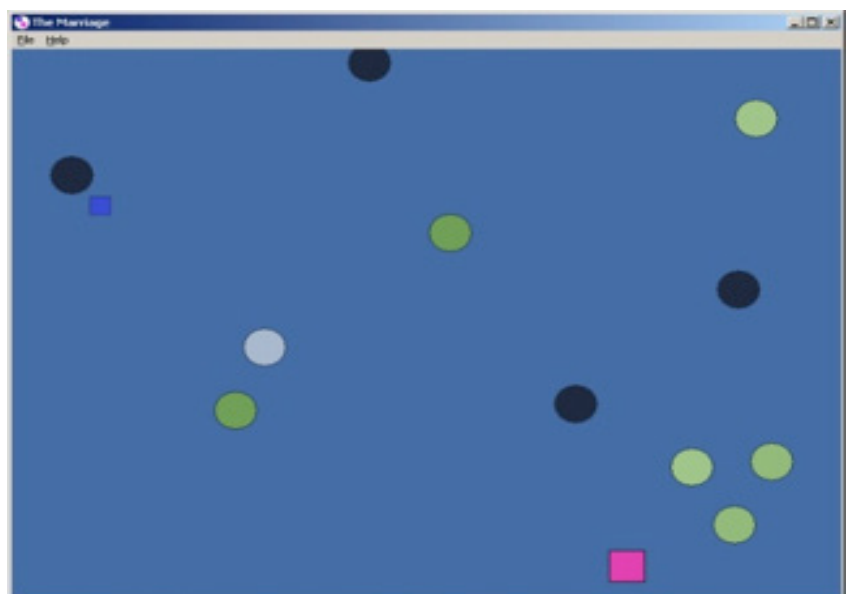

Figure 3: Objects in The Marriage are symbolic fiction-signs.

As with the other examples, the objects here operate as symbolic rules-signs: there is no relation between their form and their function. However, these objects do function as fiction-signs as well. According to Rod Humble, the pink square represents the female in the marriage, and the blue square, the male. What differentiates these signs from chess pieces is that they are symbolic fiction-signs: the relationship between their form and what they represent is arbitrary. (Note that while the common use of blue and pink to represent male and female is culturally encoded, it is still arbitrary.) As such, The Marriage is an abstract game. 
To sum up: chess is not an abstract game because the majority of its objects function as iconic fiction-signs. The Marriage is an abstract game because its objects function as symbolic fiction-signs, and go is also abstract because its objects do not function as fiction-signs at all.

\section{THE AFFECTIVE DIMENSION}

In this paper I am concerned with the experience of playing a game, which I will refer to as its "affective dimension." This term refers to the experience of playing a game as shaped by its formal properties. If we ask how it feels to play a game, or how it makes us feel, the question is concerned with the affective dimension. This aspect of games is not well understood, a fact that is not surprising given how difficult it is to describe and pinpoint.

Perhaps the best-known analysis of the affective dimension is provided by the MDA framework, a term which stands for Mechanics, Dynamics, Aesthetics (Hunicke et al. 2004). Hunicke et al. define these terms as follows: Mechanics "describes the particular components of the game, at the level of data representation and algorithms." Dynamics "describes the run-time behavior of the mechanics acting on player inputs and each others' outputs over time." Aesthetics essentially refers to what I have labeled the affective dimension, and "describes the desirable emotional responses evoked in the player, when she interacts with the game system." From the designer's perspective, the game mechanics give rise to the system's dynamics, which leads to "particular aesthetic experiences." The player views this process in the opposite direction: "aesthetics set the tone, which is born[e] out in observable dynamics and eventually, operable mechanics" (Hunicke et al. 2004). Within this framework, the aesthetic experience is determined by the game mechanics, and because of this it is possible to design for certain experiences. 
The affective dimension is meant to be an open, wide-ranging term describing a general phenomenon that can be analyzed in a variety of ways, not just via MDA. For example, similar work in describing the affective dimension (though not referred to as such) has been done by Aki Järvinen (2009), who has written extensively on how player emotions are connected to in-game goals:

As we have seen, emotions have to do with planning and goals. So does game play. Games are systems which facilitate "safe" planning towards goals, and thus they also produce various eliciting conditions for emotions.... When we are talking about player emotions, we are talking about players' appraisals and actions in relation to goals.

During a game, players experience emotions based on the status of their current goals. Because game goals are part of the game design, it is possible to design a game with the intention of eliciting certain emotions (although whether the player finds said emotions enjoyable is another question altogether). As an example Järvinen (2009) offers Missile Command (Atari 1981):

[T] he player defends six cities from incoming missiles, and has to make conflicting decisions of which cities to protect and which to leave destined for destruction, as the frequency of the missiles increases. The feeling of playing the game is often described as being characterized by panic, as one has to make quick decisions in relation to which component-of-self (a city) to prioritize in protecting, i.e. which parallel goal to abandon and which one to keep on pursuing.

Parallel goals are goals of equal value. In the game, each city is equally important, so the goal of protecting one city is just as important as the goal of protecting any other city. In this example, a primary emotion 
felt by players is panic, which results from the fast reactions necessary to play the game, combined with the absence of prioritization: they must react to everything equally quickly and give everything equal priority. This panic is part of the game's affective dimension, which is a result of the game's goal structure, a formal property of the system.

\section{METAPHOR}

Before providing examples of how experiential metaphors can work for abstract games criticism, I must first discuss the underlying processes that enable such criticism to function.

In this paper, I will be using metaphor in the sense employed by George Lakoff and Mark Johnson (1980). For Johnson (1987), metaphor is "a process by which we understand and structure one domain of experience in terms of another domain of a different kind"; metaphor is about understanding one thing in terms of another. In this context, metaphorical projection occurs when the player finds meaning in a game by analyzing how the experience of playing it is similar to another experience, thus enabling a deeper understanding of both. This projection is made possible by structural similarities between the two. While interpretation is an act of the player, and thus cannot be perfectly predicted, it is important to note that the formal properties of the game are essential to this process. Metaphorical projection is not about associating disparate objects or systems at will, but relies on systemic correlations.

\section{Structural Metaphors and Image Schemata}

Experiential metaphors belong to a class of metaphor that Lakoff and Johnson (1980) refer to as structural metaphors. These metaphors are "grounded in systematic correlations within our experience" and enable us "to use one highly structured and clearly delineated concept to structure another." The emphasis here is on structural similarities between the source and target domains that facilitate our understand- 
ing of the target. As an example, Lakoff and Johnson (1980) offer the RATIONAL ARGUMENT IS WAR metaphor, which as a structural metaphor "allows us to conceptualize what a rational argument is in terms of something that we understand more readily, namely, physical conflict." They also show how war and rational argument have structural similarities: both can be won or lost through a series of attacks, counterattacks, and defenses. Both involve intimidation, threats, claiming authority, challenging authority, insults, bargaining, and even flattery. Because of these common elements, we are able to connect war and rational argument via metaphorical projection, and this projection directly influences how we conceptualize rational argument.

Metaphorical projection is made possible by what Johnson (1987) refers to as image schemata. These are cognitive structures that organize our experience and comprehension, perhaps best explained through an example. Consider the act of cooking: cooking is a general set of actions, the specifics of which depend on what exactly is being prepared. A person cooking may be using an oven to bake a cake, a microwave to make soup, or a stovetop to prepare eggs. While "cooking" describes a wide range of possible actions and activities, these are all similar enough to fall under the same general term. Cooking, then, is a high-level image schema, and the general nature of the term is important: "cooking" does not automatically mean any one specific thing. In Johnson's (1987) view, image schemata are a fundamental component of our cognitive processes. He writes:

The view I am proposing is this: in order for us to have meaningful, connected experiences that we can comprehend and reason about, there must be a pattern and order to our actions, perceptions, and conceptions. A schema is a recurrent pattern, shape, and regularity in, or of, these ongoing ordering activities. ... I conceive of them as structures for organizing our experience and comprehension. 
Image schemata are inherently flexible and dynamic. Because of this, a given schema can be used to structure numerous similar experiences, thus enabling metaphorical projection from one experience to another. As an example, Johnson (1987) offers an analysis of the "from-to" schema. This schema is much simpler than the cooking schema, and thus can structure many disparate experiences, including cooking. This schema consists of three elements: an origin point, a terminal point, and a vector delineating a path from the origin to the terminus. Johnson argues that this schema manifests in numerous events, including: "(a) walking from one place to another, (b) throwing a baseball to your sister, (c) punching your brother, (d) giving your mother a present, (e) the melting of ice into water." Each of these cases involves the "fromto" schema. The last example is metaphorical, as the water does not actually move from one point to another; rather, the origin and terminal points are metaphorically projected onto the origin and terminal states. Structural metaphors involve comparing the structured nature of one experiential domain with that of another via an image schema.

Image schemata are significant for interpreting games metaphorically not only because they make metaphorical projection possible, but because they show how such projection relies on structural similarities between the source and target domains. Understanding one domain in terms of another is not an arbitrary cognitive act, but relies on the relevant image schemata. Image schemata necessarily shape how formal game elements can be interpreted metaphorically.

\section{Experiential Gestalts}

Gestalts are a key facet of how image schemata and metaphorical projection function. A gestalt is a "complex of properties occurring together [that] is more basic to our experience than their separate occurrence" (Johnson 1980). For example, "jumping" is a gestalt in that we conceive of the activity as a whole, not as the constituent parts that comprise a jump (applying force to the ground, losing contact with the ground 
for a period of time, then falling back down and reconnecting with the ground). Breaking down a gestalt as I have just done "will destroy the meaningful unity that makes it the particular gestalt that it is" (Lakoff and Johnson 1987). If, instead of writing "jump," I had listed the various components of jumping, it is unlikely anybody would understand what I was trying to convey; we conceive of gestalts as wholes and are generally unconscious of the constituent parts. As such, the whole is a more basic unit to our understanding than the parts.

In this paper, I am focusing on a particular class of gestalt known as an experiential gestalt. An experiential gestalt is a collection of elements or attributes that characterize an experience and allow us to comprehend that experience as a structured whole. Lakoff and Johnson (1980) elaborate:

Understanding a conversation as being an argument involves being able to superimpose the multidimensional structure of part of the concept WAR upon the corresponding structure CONVERSATION. Such multidimensional structures characterize experiential gestalts, which are ways of organizing experiences into structured wholes. In the ARGUMENT IS WAR metaphor, the gestalt for CONVERSATION is structured further by means of correspondences with selected elements of the gestalt for WAR. Thus one activity, talking, is understood in terms of another, physical fighting. Structuring our experience in terms of such multidimensional gestalts is what makes our experience coherent.

Experiential gestalts combined with image schemata are what allows us to understand one experience as being similar to another. Because experiential gestalts are structured wholes, image schemata enable us to determine when two experiences share a gestalt. This process is key to interpreting a game's affective dimension metaphorically: the gestalt of 
playing a game may be similar to that of another experience, a process known as an experiential metaphor (Rusch 2009).

\section{Experiential Metaphors}

It is possible for the affective dimension of a game to closely align with another, unrelated experiential gestalt. Doris Rusch (2009) has referred to such instances as experiential metaphors, a term referring to "the phenomenon of understanding a gameplay experience as a physical visualization of abstract ideas such as emotional processes or mental states." An experiential metaphor is a structural metaphor wherein both the source and target domains are similar experiential gestalts; Rusch emphasizes the affective aspect of an experience, rather than its structure alone. As an example, she offers a sequence from God of War II (SCE Santa Monica 2007) in which the player traverses a chasm via a grappling hook that must be attached to a series of specific points. Rusch (2009) relates the experience of playing this section to that of a transition in one's life:

By affording the player to enact courage to let go of a safe but unsatisfying status quo in order to move on to a more promising state it evokes associations to a range of similarly structured experiences. The reluctance to let go, the exhilaration of the free fall as a moment ripe with possibilities but without security, the panic that makes one latch back to the starting point, the anguish that comes with the realization that it is too late to go back, to the feeling of triumph and relief when the adventure has come to a successful conclusion-all these elements can also characterize various experiences of transition and change.

Rusch is mapping similar experiences from the source domain (life transitions) to the target domain (God of War IIs grappling-hook sequence). It should be noted that the core mechanic in the God of War II sequence 
enables Rusch's experience: the player must time letting go from one grip point and connecting to the next, risking disaster in between. For Rusch this closely aligns with the transition gestalt, which also is characterized by alternating moments of stability and uncertainty.

\section{METAPHOR AND THE SIMULATION GAP}

While the previous section describes how experiential metaphors function, merely identifying two experiences as being similar is insufficient as a critical method. To show how experiential metaphors can function in criticism, I would like to introduce and elaborate on Bogost's (2006, 2007) notion of the "simulation gap." The simulation gap is relevant here because it focuses on the relationship between a simulation (which can be a game) and another system, which is similar to the relationship between a game's affective dimension and another experience.

Gonzalo Frasca (2003) defines a simulation as follows: "to simulate is to model a (source) system through a different system which maintains (for somebody) some of the behaviors of the original system.” By this definition, some games are simulations (The Marriage), while some are not (Tetris). While I am borrowing Frasca's definition, I would like to include the notion of communication: the simulation must communicate to the player that it is based on another system in some manner. This is an essential clarification, as I will be discussing games that were not based on a source system but can be interpreted as being similar to an experience that is otherwise not intentionally related to the game.

As I have noted above, simulations enable a specific method of interpretation known as the simulation gap. The simulation gap describes the space between the simulation, the source system on which the simulation is based, and the user. This gap enables the player to perform a comparative analysis between the game and the system upon which it is based. It also allows the designer to express something about the source system by highlighting or removing certain attributes of the source. 
Bogost (2006) has defined a simulation as "the gap between the rule-based representation of a source system and a user's subjectivity" and has further written that "the ontological position of a videogame (or simulation, or procedural system) resides in the gap between the rule-based representation and player subjectivity; I called this space the 'simulation gap'" (2007). I would like to add the source system to this model. (Although it is not stated explicitly, many of Bogost's examples include the source system implicitly). This formulation is shown in Figure 4 .

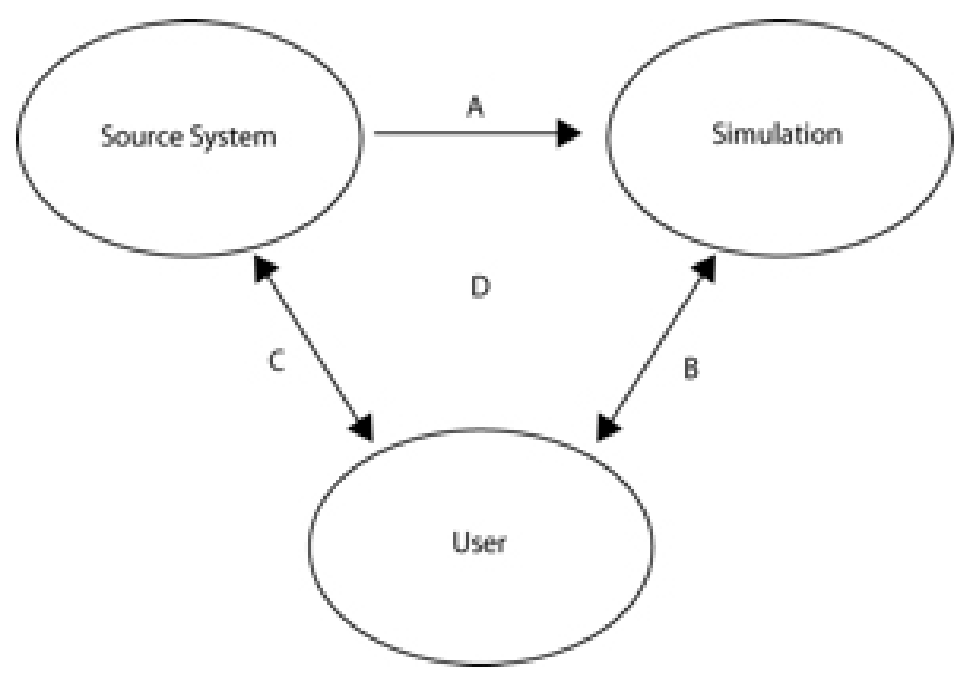

Figure 4: The simulation gap is located at point $D$, between the source system, simulation, and user.

This diagram models the interplay between the source system, the simulation, and the user. Arrow A represents the abstraction process of creating the simulation based on the source system, which involves selecting the elements of the system to include within-and exclude from-the game. Arrow B represents the user's interaction with the system, while arrow $C$ represents the user's interaction and familiarization with the source system. For example, if we are to play a game such as SimCity 2000 (Maxis 1993), we are both interacting with the 
simulation and comparing it with our knowledge of the source system, i.e., a real city. The simulation gap is located at point $\mathrm{D}$, in the space between the three elements of the system. Through interactiong with the simulation, the player compares the simulation with the source system, focusing on what the simulation has abstracted out and what it has emphasized; this then leads to an interpretation of the simulation. The user's own subjective position is a key element in how the simulation gap facilitates meaning-making: different people will attach different meanings to what the simulation includes and excludes. This also allows the player to develop a deeper understanding of both the simulation and the source system.

Furthermore, I am assuming that the simulation communicates to the player the fact that it is a simulation. This is usually done via the game's fictional elements, but can also occur via paratextual cues such as the game's title, rule book, help files, or explanatory Web sites. While the word "simulation" tends to evoke complexity, for my purposes communication is far more important. Thus a complex game like SimCity 2000 is a simulation, but so is the relatively simple September 12th (Newsgaming.com 2003), Both are based on source systems - a city and the United States government's militaristic response to the events of September 11, 2001, respectively. One can imagine a simulation that is abstract and does not inform the user that it is a simulation. And while such a simulation would still qualify as a simulation, I will not be taking such examples into account because such a game would be difficult to identify as a simulation and thus could not rely on the simulation gap to shape meaning. Under my definition, then, Tetris is not a simulation because it does not communicate a source system.

Interpreting a game via metaphorical projection and via the simulation gap involves two similar yet distinct cognitive acts. In the case of a simulation, the player is presumably aware that the game is based on 
a source system, and begins playing with the simulation gap already in place. The player is then able to contrast the simulation with the source as play progresses.

Interpreting a game as an experiential metaphor, however, generally occurs in one of two ways. It can result from a reflective process that requires a close analysis of the game's affective dimension, or can occurspontaneously and intuitively during the play of game. The key difference lies in when and how the player connects the game to the outside system or experience. In the case of simulation, the player is given a source system before play even begins, while metaphorical projection occurs during and after play. However, in both instances a player can interpret the game as expressing ideas or making claims about the other system or experience by how it highlights or de-emphasizes its various elements.

I have noted above that the player's comparison of the simulation with the source system can lead to a deeper appreciation of both. Although the initial process is different- the player is not given a source system - experiential and structural metaphors allow the player to compare the game with another experience, system or idea in a manner similar to that of the simulation gap. This is possible because both metaphorical projection and the simulation gap necessarily amplify and diminish various aspects of the system or idea connected to the game. In the case of simulations, the abstraction process involves choosing which elements of the source system to include and which to exclude. Lakoff and Johnson (1980) note that a similar phenomenon occurs when we understand something metaphorically:

In allowing us to focus on one aspect of a concept (e.g. the battling aspects of arguing), a metaphorical concept can keep us from focusing on other aspects of the concept that are inconsistent with that metaphor. For example, in the midst of 
a heated argument, when we are intent on attacking our opponent's position and defending our own, we may lose sight of the cooperative aspects of arguing.

Thus, understanding a game as a metaphor for something else is very similar to understanding a game as a simulation. In both instances, we are able to find meaning and expression in the differences. To return to Rusch's (2009) God of War II example, Rusch notes that failure to swing from one point to the next results in the player's death. While this aspect makes the affective experience more intense, understanding the sequence metaphorically masks the importance of death in the game because it does not correlate with any elements of the transition gestalt.

\section{A CRITICAL METHOD}

From these concepts of metaphor and simulation, it is possible to derive a set of methods for the metaphorical interpretation of the affective dimension. Analyzing how an abstract game functions as an experiential metaphor involves the following process: isolating the key elements in the game's experiential gestalt, analyzing how those elements are tied to a common sequence of states within the game, and identifying emotions that arise from those states. From there it is possible to identify a similar, more general experiential gestalt. We can then link the two gestalts through metaphorical projection by mapping elements from the general gestalt (the source domain) to the game's gestalt (the target domain). In this section, I provide two examples of abstract games functioning as experiential metaphors.

\section{Tetris as an Experiential Metaphor}

The best-known example of an interpretation of the affective dimension via metaphorical projection is Janet Murray's (1997) interpretation of Tetris. As noted above, Tetris is an abstract game because its objects - the falling blocks — do not function as signs within the game's fiction; the game has no fiction at all. 
This game is a perfect enactment of the overtasked lives of Americans in the 1990s_ of the constant bombardment of tasks that demand our attention and that we must somehow fit into our overcrowded schedules and clear off our desks in order to make room for the next onslaught.

For Murray, the source domain is the "overtasked lives of Americans in the 1990s" and the target domain is Tetris: she is projecting aspects of the source onto the target, thus forming her interpretation.

Scholars and critics have offered numerous responses to this interpretation. Markku Eskelinen (2001) has referred to it as "horrid," because "instead of studying the actual game Murray tries to interpret its supposed content, or better yet, project her favourite content on it; consequently we don't learn anything of the features that make Tetris a game." Eskelinen's reaction is interesting because he seems to be confusing intent: he himself says that she is trying to interpret the game, whereas he is interested in the game's formal properties. Clearly, their goals are different; and one approach does not automatically invalidate the other.

Ian Bogost has reacted more positively to Murray's interpretation, calling it "endearing" (2006) and claiming that it is "entirely reasonable," in that she "offers something essential: evidence from the work itself" (2009). However, he claims that Murray wants the game to "function only narratively" (2009). While I cannot speak to Murray's intentions, as it stands her interpretation of Tetris is metaphorical, not about reading narrative into the game. She is mapping elements from one domain of experience onto another, not arguing that the game tells a story or relates specific events.

However, Bogost (2007) has also criticized Murray's interpretation for 
its lack of precision:

Janet Murray's interpretation of the game as a representation of the unfettered demands of global capitalism would become much more comprehensible to the uninitiated player if she explicitly correlated the game's unit operations with the real world characteristics she has in mind. For example, the constant bombardment of tasks is correlated to the continuous generation of new blocks, and the need to fit unending work into overcrowded schedules and desks correlates with the completed lines which disappear, but only to give way to another onslaught of work.

The correlations Bogost seeks through unit operations are effectively mappings from the source to the target domain. The experience of receiving an endless number of new tasks is metaphorically projected onto the experience of receiving an endless number of new blocksboth of which demand attention. By pushing this type of metaphorical analysis farther, we can see how effective the interpretation is.

From this example it is clear that Murray's interpretation of Tetris is as an experiential metaphor, as Rusch (2009) notes. To evaluate Murray's interpretation more closely, we must begin by examining which experiences in the source domain map to which game states in the target domain, a task similar to Bogost's correlations between the game's unit operations and the real-world system. As I have noted, the source domain is the "overtasked lives of Americans in the 1990s" and the target domain is Tetris. The source domain is unfortunately vague, but we can infer that Murray specifically means Americans employed in some manner of white-collar occupation, by her references to desks and schedules. The first relevant experience is that of an impending task, which in a white-collar job could be any number of things. In 
Tetris this maps to a game state in which a new falling block has just begun descending (the state of the rest of the game does not affect this particular mapping). In both instances there is emotional tension originating in the uncertainty of the outcome, because the quality of the completed task has lasting effects. In Tetris, poor block placement will lead to future game states that are difficult to manage, while in the workplace poor performance will have short- and long-term negative effects; in both instances, this leads to anxiety and stress. Finally, the game reaches a state such that a line is cleared, which leads to a brief period of relief that is soon interrupted by the next block. This sequence of states maps to a sequence of experiences characteristic of the source domain: completing a task brings a brief respite, which is inevitably interrupted by a new assignment, which in turn brings back the previous anxiety.

Murray's (1997) reading of Tetris is effective in that she has identified how the experiential gestalt of playing the game- the affective structure of the experience that results from the sequence of game statesaligns with the experiential gestalt of white-collar employment. Both gestalts consist primarily of tension, uncertainty of outcome, consequences, and temporary relief. For Murray, the affective dimension of Tetris contains a deeper meaning: the game encourages reflection on white-collar employment. It can also be interpreted as expressing frustration with such employment: the inability to "win" at Tetris maps to the phrase "dead-end job," meaning an occupation without opportunity to advance.

\section{Tipping Point}

Another example of an abstract game that functions as an experiential metaphor is Tipping Point (2009), a cooperative board game1 developed by a team of students working in the Singapore-MIT GAMBIT Game Lab; I served as producer and designer on the team. It is a simulation of product development cycles in a corporate environment. 
The game is a relevant example because it is an abstract simulation, and can be interpreted as an experiential metaphor of balancing work over a school semester.

The game is based on a simple model of product development derived from the research of Repenning et al. (2001), in which some projects are in "concept development" while others are in "product design and testing." In the game, each player is managing one or more projects, which are represented by the colored crosses in Figure 5. The colored hexagons are a player's production work tokens, and the black circles with white exclamation marks are concept work tokens (no tokens are in play in this figure). Thus the game is abstract: the objects (production and concept tokens) are symbolic fiction-signs.

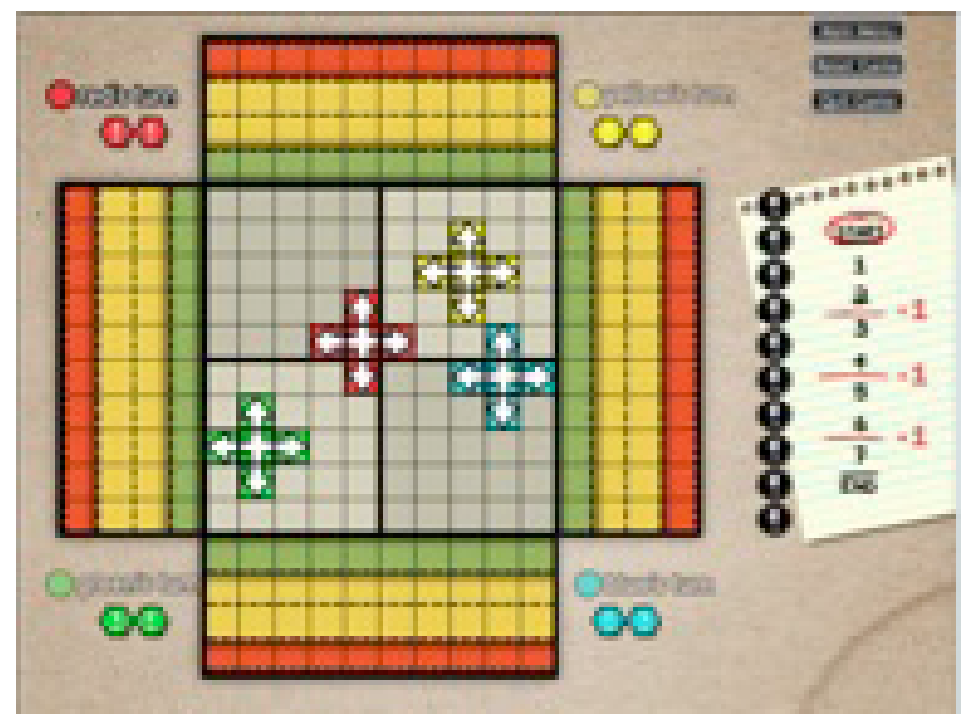

Figure 5: An initial state of Tipping Point.

After a player's turn, all of his or her projects grow one square in each orthogonal direction. Players must work together to prevent projects from growing onto the red squares at the edges of the board; failure to do so results in a loss for everyone, not just the owner of the project. 
Players complete projects by placing concept and production work tokens to prevent the projects from growing; a project that cannot grow is considered completed. On a player's turn he or she may place both of their production tokens, or one of the concept tokens from the communal pool, on the board. Production tokens stay on the board for only one round, whereas concept tokens remain indefinitely. When a project is completed, its owner must then place a new project, and the group earns one point. The players must earn eight points to win, but after every two points they take on an additional project. This means that at the start of the game only four projects will be on the board at a time, but at the end there will be seven. The increased number makes the game significantly more difficult, as projects that grow into each other combine to form a single project; these compound projects then grow faster and are harder to complete.

The simulation thus emphasizes the balance of concept work and production work. Production work represents last-minute "firefighting" or "crunch" work: while it has a greater short-term benefit than the concept work (because two points may be blocked on a turn instead of one), this benefit disappears on the player's next turn. Concept work's permanence represents the ways that effective planning early in the development process has long-term benefits that last beyond the current project: placing concept tokens always makes the game easier later on, and players will often find themselves in a situation where it is impossible to complete a project without them. The game makes a strong argument in favor of planning; this was a conscious design goal.

While the game design assumes four players, Tipping Point is equally playable with fewer, even one. With respect to the solo version of Tipping Point, one possible metaphorical interpretation of the affective dimension is as an experiential metaphor for managing coursework over a semester. As I have previously noted, metaphorical projection necessarily amplifies and diminishes various aspects of each domain. 
In the case of Tipping Point, understanding the game as an experiential metaphor for a school semester amplifies the planning and coordination aspects of managing coursework, and diminshes the nature of the work done on projects. The experience of researching and writing a paper does not map to any element in the game, but scheduling and planning map very closely to Tipping Point's core mechanic: deciding what type of work to do, when to do it, and where to apply it. In the solo version the player takes the turn of each of the four colors. In metaphorical terms, each color maps to a different class: each has its own assignments that must be completed by different deadlines. In the game, for example, the red project may reach the red zone in four turns, whereas the blue project will reach it in three. While the game's initial state is semi-random (each project begins in a random square of a different quadrant), it is characterized by slight apprehension. In this state the projects are generally far away from their deadlines, but the player is aware that the deadlines will approach very rapidly. This state maps to the experience of looking at syllabi during the first week of class. At that point the semester is not particularly stressful, yet the knowledge that the deadlines are already approaching leads to a similar feeling of apprehension.

Over the first few turns of play, the state changes significantly: projects begin approaching their deadlines, and the player begins placing various work tokens. Concept tokens create game states in which very few projects are blocked, but the short-term disadvantage quickly changes to a long-term advantage as concept tokens assist in finishing multiple projects over time. A state in which the board is heavy on concept tokens maps to the experience of having invested time in general academic work, such as improving one's writing or developing one's research interests. In both cases there is a sense of initial futility, as these efforts have less of a direct impact on the completion of single projects or assignments, but this frustration is gradually replaced by appreciation as the long-term benefits become apparent: as with 
concept tokens, this type of work has benefits across several assignments over time. A game state in which numerous production tokens have been placed maps to the experience of having spent time on tasks related to a specific assignment, such as formatting or proofreading a paper. These tasks are necessary to complete the assignment but are not particularly useful elsewhere. Such work can bring some relief, in that it usually means a task is nearing completion, but this relief is accompanied by the sense that the time could have been better spent on more fruitful pursuits.

In Tipping Point, and during a semester, completed projects or assignments are immediately replaced by new assignments. This leads to a state in which the new projects are relatively far from their deadlines, which in turn leads to a brief sense of relief: there is now time to place more concept tokens, which will make the game easier later on. Such a state maps to the relief one feels after handing in an assignment and then having time to focus on more general projects, such as reading or attending to nonacademic tasks.

While this is similar to the experience of working on product development for a company, the key difference is in the ramp-up of work and the associated affective experience. Repenning et al. (2001) assume that a given company is always producing two products at once with no ultimate endpoint, whereas Tipping Point and a school semester are characterized by the increase in the number of simultaneous projects over a set period time. As the game gets closer to the end, the greater number of projects leads to stronger feelings of tension, apprehension, and panic. The same is true of a semester.

Tipping Point ends with a sort of climactic implosion: the final project is often an enormous, threatening mass that is completed all at once, leaving behind a few smaller projects that must be cleaned up but are no real threat. This sequence of states at the end of the game maps to 
the experience of a week of final exams, especially when several are scheduled on the same day. After the most intimidating final papers or tests are completed, assignments of lesser concern often remain. At this point the game/semester is much easier, and the remaining tasks seem almost trivial in comparison with the feats just accomplished. The mappings I have described allow the affective dimension of Tipping Point to function as an effective experiential metaphor for progressing through a semester, as both have similar experiential gestalts. Interestingly, the rhetorical point of Tipping Point as a simulationthat planning and conceptual work are essential for success—also applies to Tipping Point as a metaphor: the key to success in dealing with multiple tasks is effective long-term planning. However, I would argue that the game is more effective as an experiential metaphor than as a simulation, largely because of the ramp-up in work over time that is followed by the sudden cessation of new projects. (This was a design decision intended to make the game more engaging.) The sequence of states that results has more in common with a school semester than with a product-development cycle, which means that the affective experience of playing is closer to the experiential gestalt of a semester as well.

\section{CONCLUSION}

As I have shown in the above examples, experiential metaphors provide an effective means of criticism for abstract games. Using this method, it is possible for an interpreter to create meaning out of a game that, on the surface, seems to be lacking any type of expression or meaning beyond the game itself.

I want to emphasize that understanding how to locate meaning in abstract games is of paramount importance to understanding the strengths and potentials of games as an expressive medium. Abstract games are quite possibly the primordial game configuration; only recently have characters and stories become possible. Consequently, 
any general theory of how games can express and communicate ideas must be applicable to abstract games. If such a theory is incompatible with abstract games, then it cannot be about games specifically. Furthermore, as Rusch's (2009) example shows, experiential metaphors are equally applicable to more representational games that include rich fictional worlds and characters. Thus, experiential metaphor is an ideal method of criticism, as it is applicable to many different types of games. Lastly, this critical methodology implies a path for design research. If an abstract game is designed such that the affective dimension is an experiential metaphor, and the game provides no clue as to what that metaphor might be, will other players connect the experience as intended? This certainly seems possible, as evidenced by the fact that I am able not only to understand Murray's (1997) metaphorical interpretation of Tetris, but also to identify the elements of the source domain and how she has mapped them onto the target domain. This implies that abstract games consciously designed to function as an experiential metaphor can be understood by a broad audience. In this case, metaphor-based game design offers enormous potential for creating games of all kinds that are meaningful and expressive in a novel way.

\section{ACKNOWLEDGMENTS}

Thanks to Doris Rusch for her frequent feedback on this work. Thanks as well to the many participants in the former Singapore-MIT GAMBIT Game Lab research meetings in 2010-2011 for their invaluable comments and insights.

\section{ENDNOTES}

1. For clarity's sake, when Lakoff and Johnson refer to a metaphysical concept it is printed in capital letters. I have continued this convention for similiar reasons.

2. The game was later implemented in Flash, and is currently playable online at <http://gambiut.mit.edu/loadgame/tippingpoint_digital. php> 


\section{REFERENCES}

Atari. Missile Command.[Atari 2600], Atari, 1981.

Bogost, Ian. Unit Operations. Cambridge: MIT Press, 2006.

Bogost, Ian. Persuasive Games. Cambridge: MIT Press, 2007.

Bogost, Ian. "Puzzling The Sublime." Gamasutra, Dec. 23, 2009. $<$ http://www.gamasutra.com/view/feature/4225/persuasive_ games_ puzzling_the_.php $>$. Accessed 1/30/09.

Chandler, Daniel. Semiotics: The Basics. 2nd edition. Routledge: New York, 2007.

Eskelinen, Markku. “The Gaming Situation.” In Game Studies, vol 1, issue 1, July 2001. <http://www.gamestudies.org/0101/eskelin en/>.

Frasca, Gonzalo. "Simulation versus Narrative: An Introduction to Ludology." In The Video Game Theory Reader, ed. Mark J. P. Wolf and Bernard Perron. New York: Routledge, 2003.

Humble, Rod. The Marriage. [Windows PC], 2007. <http://www.rodvik.com/rodgames/marriage.html>.

Hunicke, Robin; LeBlanc, Marc; and Zubke, Robert "MDA: A For mal Approach to Game Design and Game Research.” In Pro ceedings of the AAAI Workshop on Challenges in Game AI, 2004: 04-04. <http://www.cs.northwestern.edu/ - hunicke/ MDA.pdf>.

Järvinen, Aki. "Games Without Frontiers: Theories and Methods for Game Studies and Design.” Ph.D. Thesis, University of Tam pere, Finland, 2008. <http://acta.uta.fi/english/teos php?id $=11046>$.

Järvinen, Aki. "Understanding Video Games as Emotional Experiences." In The Video Game Theory Reader 2, ed. Perron and Wolf. New York: Routledge, 2009.

Singapore-MIT GAMBIT Game Lab. Tipping Point.[PC] 2009. $<$ http://gambit.mit.edu/loadgame/tippingpoint_digital.php>. Johnson, Mark. The Body In The Mind. Chicago: The University of 
Chicago Press, 1987.

Juul, Jesper. Half-Real. Cambridge: MIT Press, 2005.

Lakoff, George, and Johnson, Mark. Metaphors We Live By. Chicago:

The University of Chicago Press, 1980.

Maxis. SimCity 2000. [PC], Maxis, 1993.

Murray, Janet. Hamlet on the Holodeck: The Future of Narrative in

Cyberspace. Cambridge: MIT Press, 1997.

Newsgaming.com. September 12. [PC], Newsgaming.com, 2003.

$<$ http://www.newsgaming.com/games/index12.htm>.

Nintendo. Super Mario Bros. [Nintendo Entertainment System], Nin tendo, 1985.

Pajitnov, Alexey. Tetris [Multiple], 1984.

Peirce, Charles S. Essential Peirce: Selected Philosophical Writings. Vol.

2. Ed. Nathan Houser and Christian Kloesel Bloomington:

Indiana University Press, 1998.

Repenning, Nelson P., Gonçalves, Paulo, and Black, Laura J. "Past the

Tipping Point: The Persistence of Firefighting in Product Devel opment." California Management Review. Vol.43, 2001.

Rusch, Doris. "Mechanisms of the Soul: Tackling The Human Condi tion in Videogames." In proceedings of DiGRA 2009, Breaking New Ground: Innovation In Games, Play, Practice, and Theory. London: Brunel University, 2009. <http://www.digra.org/dl/ display_html?chid=http://www.digra.org/dl/db/09287.01371. pdf>.

SCE Santa Monica. God of War II. [Playstation 2], SCEA, 2007. 
Transactions of the Digital Games Research Association Vol. 1 No. 1, ISSN 2328-9414, June 2013

(C) The text of this work is licensed under a Creative Commons Attribution -- NonCommercial --NonDerivative 2.5 License (http://creativecommons.org/licenses/by--nc-$\mathrm{nd} / 2.5 /$ )

IMAGES: All images appearing in this work are property of the respective copyright owners, and are not released into the Creative Commons. The respective owners reserve all rights. 


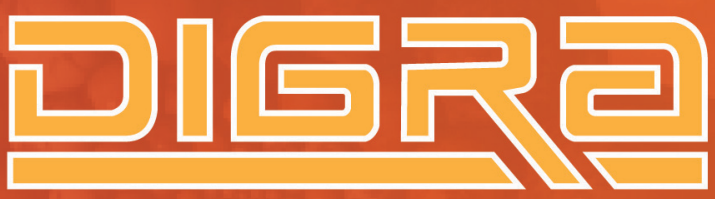

Transactions of the Digital Games Research Association

Volume $1 \quad$ Number 1 June 2013 